\title{
Improving TFRC Performance against Bandwidth Change during Handovers
}

\author{
Dagang Li, Kristof Sleurs, Emmanuel Van Lil and Antoine Van de Capelle \\ TELEMIC, Dept. of Electrical Engineering-ESAT, Katholieke Universiteit Leuven \\ Kasteelpark Arenberg 10, B-3001 Heverlee, Belgium \\ Email: dagang.li@esat.kuleuven.be
}

\begin{abstract}
TCP-Friendly Rate Control (TFRC) is a congestion control mechanism that can compete fairly with TCP while maintaining a much smoother transmission rate. The smoothness is achieved at the cost of responsiveness, which makes it slow to settle down when handed-over to a different network. In this paper we will study how to improve the TFRC performance when the available bandwidth is changed during a handover. An enhancement is proposed that can eliminate most of the heavy packet loss during a bandwidth-decreased handover. The definition and conditions of a smooth TFRC handover is also provided. The improvement over the original TFRC is proved with simulation results.

Index Terms-TFRC, Handover, Congestion Control
\end{abstract}

\section{INTRODUCTION}

The success of today's gigantic Internet is largely due to its stability which depends on the end-to-end congestion control provided by the dominant transport protocol TCP. TCP supports reliable data delivery service very well, but it is not suited for real-time applications with timeliness requirement. The highly variable transmission rate of TCP is also not desirable for applications that require a smooth throughput. TCP-Friendly Rate Control (TFRC) [1] is a congestion control mechanism proposed for these applications. It does not retransmit broken or lost packets, so the following packets will not be delayed; it has a much lower variation of throughput over time compared with TCP; furthermore, it competes reasonably fair with TCP, aka it is TCP-friendly. The fairness comes from the fact that TFRC uses TCP throughput equation to calculate its sending rate, as discussed later in the next section.

The penalty of smoothness is responsiveness: TFRC responds slower than TCP to the changes in the available bandwidth. Clearly, it is more severe a problem during a handover, since the available bandwidth seen by a TFRC flow can change abruptly in an instant. When more bandwidth is available, TFRC takes much time to fully utilize the new bandwidth; when less bandwidth is available in the new network, excessive packets will be injected into the network before TFRC sufficiently slows down, which not only leads to more packet drops but also causes persistant congestion that affects all competing flows in the new network.

This problem has been addressed by a number of researchers. Authors of [4] proposed an explicit handover notification mechanism that resets the TFRC receiver state after a handover and sends adjusted feedback reports for 3 RTTs to reflect the bandwidth change. However the discussion lacks details and the exact algorithms for handover detection, bandwidth measurement and report adjustment are not discussed. In [6] a detailed mechanism is presented using packet-pair to detect the bandwidth change caused by a handover. When a bandwidth increase is detected, TFRC just goes back to slow start to probe for the new available bandwidth; when bandwidth decreases, an urgent message is sent to the sender with the estimated bandwidth from the detection mechanism. The performance depends on the accuracy of the estimation algorithm. On the other hand, when both the old and new link are accessable during a handover, authors of [5] propose to probe the available bandwidth of the new link before a handover is performed. This technique requires changes in both application layer and networking layer, which makes it hard to be deployed in the current Internet.

All these previous work tried to tackle the TFRC handover problem by accelerating the adaptation of TFRC to the new network: the faster the adaptation, the less the negative effects last. However, if we can directly minimize the negative effects during the handover, TFRC can be left to adapt in its natural way that persists its overall behavior. This is exactly what we will do in this paper. Because TFRC is not designed to aggressively find and use available bandwidth but to maintain a relatively steady sending rate while being responsive to congestion [2], we will focus on the case of bandwidth decrease. In the rest of the paper, we first study how TFRC reacts to a handover-induced bandwidth step-decrease in section 2 . Section 3 identifies the causes of the problem and describes an enhancement to TFRC for a smooth transition during such a handover. Simulation results are presented in section 4 to show the improvement of the proposed enhancement. Section 5 concludes the paper.

\section{TFRC PERFORMANCE IN HANDOVERS}

TFRC is an equation-based congestion control protocol. The recommended equation used in TFRC is a slightly simplified version of the throughput equation for Reno TCP from [3], as shown below:

$$
X=\frac{s}{R \sqrt{\frac{2 b p}{3}}+r t o \cdot 3 \sqrt{\frac{3 b p}{8}} p\left(1+32 p^{2}\right)}
$$

The sending rate $X$ as a function of packet size $s$, roundtrip time $R$, TCP retransmission timeout rto, loss event rate $p$ and finally $b$ which is the number of packets acknowledged 


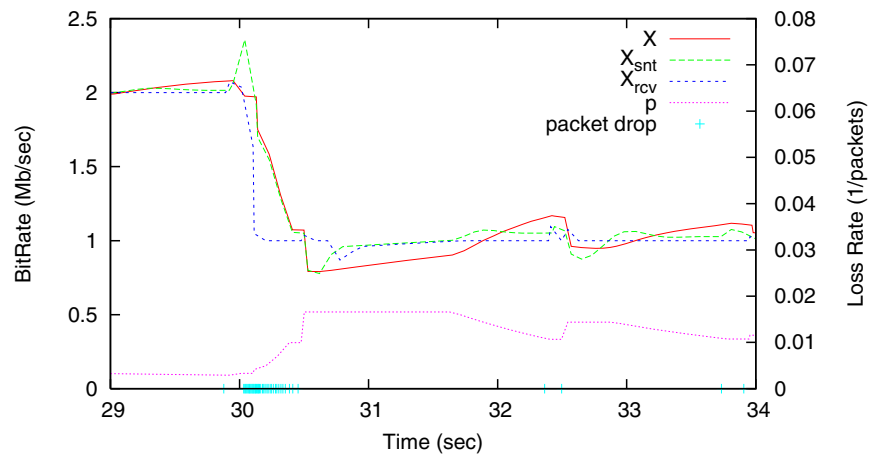

Fig. 1. TFRC during a Bandwidth-decreased Handover

by one ACK. A loss event is defined as the aggregation of all the packets lost within one RTT. Since rto is normally set to $4 R$, from Eq. 1 we can see that the only variables that might be affected by a handover are the round-trip time $R$ and loss event rate $p$. In other words, for TFRC to send with an appropriate rate after a handover, $R$ and $p$ have to be adjusted correctly. Before this is done, TFRC will keep sending at a higher rate if bandwidth available in the new network is lower, which increases $p$ and finally drags the sending rate down. (In this paper we assume RTT is not changed during the handover. Handover with changed RTT has been studied in [8]).

The difficulties of adjusting loss event rate $p$ fast and accurately lie in the fact that $p$ is an averaged variable updated using Weighted Average Loss Interval (WALI) mechanism. $p$ has a memory of the past and requires multiple samples to update during a step change. The fact that the samples cannot be collected faster than one per RTT further slows the adaptation down. It is also hard to predict an accurate $p$ in advance since $p$ is influenced by a lot of changing factors in the network. The good news is that WALI reacts to a $p$ increase (corresponding to a bandwidth decrease) much faster than to a decrease. It takes about 5 RTTs for TFRC to half its sending rate during persistent congestion [2]. The bad news is that heavy packet loss happens during this period, as shown in Fig. 1, which inevitably deteriorates the quality of the carried traffic, especially those coded streams that can not recover from contineous packet loss.

\section{A. TFRC Conservative Option}

Since we cannot get to the right $p$ immediately after the handover without several RTTs' probing and sampling, an intuitive way to reduce the heavy packet loss is to regulate the sending rate also by some other means than the equation alone, while this other regulation can reflect the abrupt change. Actually within TFRC itself, the calculated sending rate $X$ is already shaped by mechanisms like oscillation reduction $(\S 4.5$ of [1]), receiving rate control ( $\$ 4.3$ of [1]) and the maximum increasing rate (1 packet per RTT). An interesting new option called conservative _ is proposed in [7] to introduce selfclocking to TFRC. It limits the sending rate to at most the receiving rate in the previous round trip if a packet loss is detected; and in the absence of loss, the maximum sending

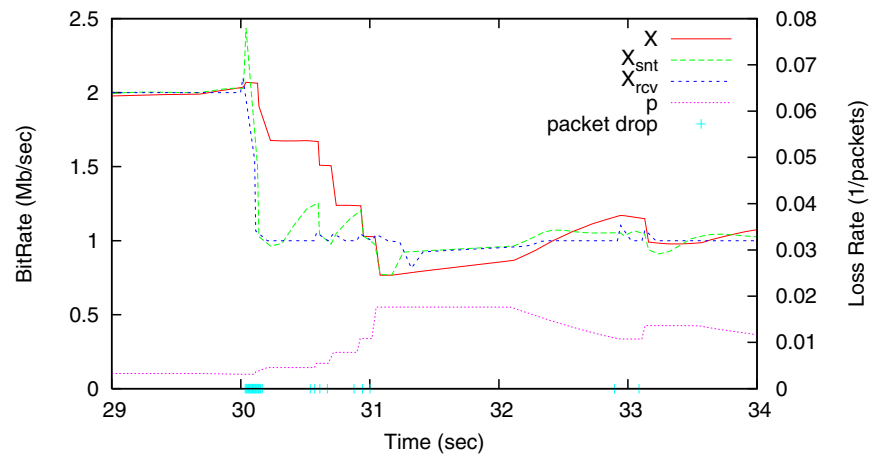

Fig. 2. Conservative TFRC during a Bandwidth-decreased Handover

rate is set to a constant $C$ times the earlier receiving rate. A conservative TFRC can react much faster to the bandwidth decrease after a handover, because the first packet loss in the new network will immediately throttle the sending rate to the new receiving rate, as shown in Fig. 2.

Although the conservative TFRC experiences much less packet loss, the loss is still not neglectable. Furthermore, this option is not mandatory, and the conservative behavior is not always desirable. For example, in the case when the new network is already fully utilized by congestion controlled flows, the handed-over TFRC flow has to compete for its fair share. The congestion experienced by this TFRC flow will be the most severe right after the handover, whileas later its share should grow as the other flows back-off. If the sending rate is limited by the previous receiving rate, TFRC will not be able to catch up towards its fair share and gets stuck with an under-partitioned share for a long time.

\section{SMOOTh TFRC HANDOVER}

In this paper we want to use the idea of self-clocking but in a different mechanism that can apply to TFRC with or without the conservative_option. It should also have a minimum packet loss and more competitive for its fair share.

One thing to notice here is that our goal is not a lossless handover but a smooth handover, because packet loss is an indispensable ingredient of TFRC congestion control ${ }^{1}$, because it is used to update the loss event rate $p$. We define a smooth TFRC handover as a handover without excessive packet loss. Because all packets lost within one RTT are considered as a single loss event, packet loss other than the first one would be regarded as excessive.

\section{A. Design Considerations}

We start with a detailed analysis on the handover of conservative TFRC, which is shown in Fig. 2. In order to find out what causes all the packet loss, the corresponding time line graph is also given in Fig. 3.

The handover happens at $30 \mathrm{sec}$, or time $t_{0}$ in Fig. 3 . We use $X_{0}$ to generally represent the sending rate before the handover.

\footnotetext{
${ }^{1}$ Of course the role of packet loss can be replaced by ECN marking, and the discussions here can be easily applied to the case of ECN as well.
} 


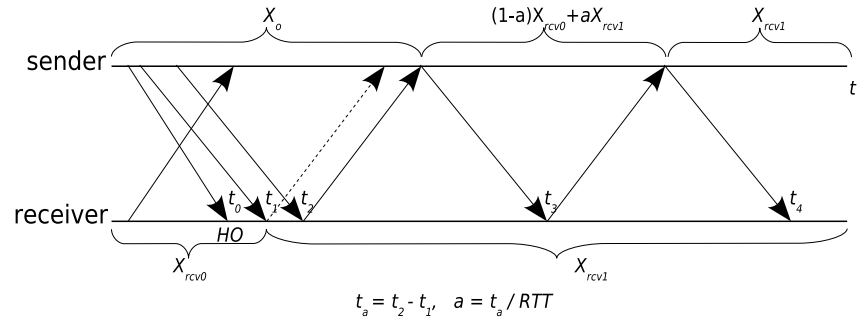

Fig. 3. Conservative TFRC during a Handover

It is reasonable to assume that $X_{0}$ is relatively stable since its variance should be small compared to the bandwidth change during the handover. At time $t_{1}$ the first packet via the new route arrives. Because the bandwidth of the new network is smaller, queue starts to build up in the bottleneck link. Finally at $t_{2}$ a packet loss is detected and an urgent ACK is sent to the sender. In this ACK the reported receiving rate is a weighted average of the old and new receiving rate, because the handover happens between it and the previous ACK. The conservative_ option immediately sets the sending rate to this reported receiving rate. Because it is still higher than $X_{r c v 1}$, which apparently equals the available bandwidth in the new network, queue building-up continues. This is the reason for the continuous packet loss seen in Fig. 2 right after the handover. Only in the next ACK at $t_{3}$ the measured receiving rate will be $X_{r c v 1}$, therefore the train of packet loss only stops at time $t_{4}$. After $t_{4}$ the normal behavior of conservative TFRC applies: a little higher sending rate is probed when no packet gets lost in the previous round, or otherwise it is set to the reported receiving rate.

From the above discussion two negative effects are identified. First, the correct receiving rate is only reported in the second ACK after the handover, which leads to a 2-RTTlong heavy packet loss period $\left(t_{4}-t_{2}\right)$. From Fig. 3 we can see that the receiver actually switches to $X_{r c v 1}$ already from $t_{1}$. If the new receiving rate can be reported to the sender from $t_{1}$, then only half a RTT later the sending rate will be correctly throttled. This will decrease the packet loss period to $\min \left(0, R T T-t_{a}\right)$.

Two actions are required for this purpose.

1) Since it is not possible to measure the receiving rate as early as $t_{1}$, we resort to the original way of selfclocking used by TCP: every packet received from time $t_{1}$ is acknowledged and the sender only sends out a new packet upon each new ACK. This way $X_{r c v 1}$ is implicitly applied to the sending rate right from the ACK sent at $t_{1}$ is received.

2) This per-packet-acknowledgement ends at time $t_{2}$, when an ACK is due to be sent out because of packet loss. In order to report the correct receiving rate $X_{r c v 1}$, packets received before the handover should be excluded. This can be done by concluding the previous round by the ACK sent at time $t_{1}$ (dashed line in Fig. 3), so the new round only contains packets received after the handover.
Second, the conservative way of probing doubles the adaptation time for $X$ compared with the case of normal TFRC. As mentioned earlier, the conservativeness will hinder the TFRC when it has to compete for its fair share after a handover.

In our proposal we use a more aggressive approach than conservative TFRC to adjust its sending rate after a handover: the sending rate is always set to 1 packet per RTT higher than the reported receiving rate, until this rate matches the theoretical $X$ calculated from Eq. 1. 1pkt/RTT is chosen so that if the sending rate overshoots, at most one packet will get lost in the next round. This is just enough to mark another loss event that decreases $X$ towards the available bandwidth, which meets the definition of a smooth handover. If no packet gets lost, another $1 p k t / R T T$ is probed.

The enhancement discussed above will be activated whenever TFRC undergoes a handover, regardless of the conservative_option, for the best performance.

\section{B. Design Details}

In this section we present some important technical details when applying the enhancement to TFRC.

1) Receiver Side: The receiver should be able to recognize the first packet arriving after a handover, which requires indications from lower layers, such as the sender of the last hop, the arriving interface, or specific marking from the network. In this paper we assume the availability of such crosslayer mechanism.

If store-and-forward is used during the handover to minimize packet loss, forwarded packets from the old network may interwine with packets routed directly to the new network. In this case the forwarded packets should be excluded from the above procedures, since they bear no information of the new path.

2) Sender Side: The sender should be able to maintain a new state for the adaptation period during a handover. In this state the proposed enhancement takes over the control of the sending rate. Oscillation reduction should be disabled in this state, because it uses RTT history to monitor queue dynamics, which is not valid any more after a handover.

\section{Conditions for Smooth TFRC Handover}

In our proposed enhancement, the packet loss period after a handover can be reduced to 0 if $R T T<t_{a}$, so no packet gets dropped before TFRC starts probing for higher sending rate. During $t_{a}$ the sending rate is always higher than the receiving rate, therefore this condition is only possible if all the excessive packets are buffered in the network. From the definition of a smooth TFRC handover, at most one packet can be lost during this loss period. Let the sending rate be $X_{0}$, we have

$$
\left(R T T-t_{a}\right) \cdot\left(X_{0}-X_{r c v 1}\right)<s
$$

Because $t_{a} \cdot\left(X_{0}-X_{r c v 1}\right)=B$, where $B$ is the required buffer size in the bottleneck link, therefore a smooth TFRC handover is possible when

$$
B>R T T \cdot\left(X_{0}-X_{r c v 1}\right)-s
$$




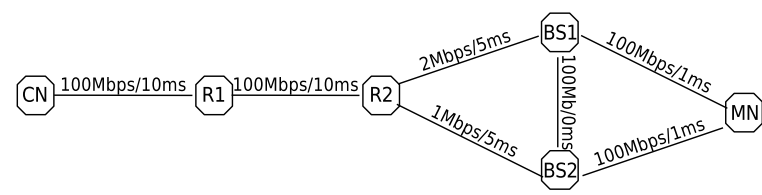

Fig. 4. Simulation Settings

If sufficient buffer is available and no packet gets lost in this period, then $t_{2}$ would be the time of the next periodic ACK which can as well end the per-packet-acknowledgement.

\section{Performance Evaluation}

The topology in Fig. 4 is used in the simulations. CN keeps sending a TFRC flow to the MN, while the latter switches from BS1 to BS2. Parameters of the links are shown in the figure. Buffer-and-forwarding is used in the simulations so all possible packet loss will be caused by the bandwidth decrease. The handover happens at $30 \mathrm{sec}$ and the simulation lasts 60 sec. Fig. 1 and Fig. 2 are also generated with these settings.

Fig. 5 shows the performance of the enhanced TFRC. With the proposed enhancement, the continuous packet loss lasts for a much shorter time compared with the original TFRC, with or without the conservative_option. The following distributed packet losses are the result of the aggressive bandwidth probing mechanism. This mechanism helps the enhanced TFRC to complete rate adaptation as fast as the original TFRC. Since these losses are distributed into different RTTs, the effect on the quality of the carried traffic should be small. If desired, the aggressiveness can also be adjusted, possibly adaptively, to meet the requirement on packet loss.

Now we want to have a smooth TFRC handover. According to Eq. 3, we come up with a necessary buffer size of at least 7 packets in the bottleneck link R2-BS2, based on the settings shown in Fig. 4. From the simulations we find out that the actual minimum buffer size would be 8 packets. The difference between the measured and the theoretical value is caused by the packetization of the buffer. The result with a 8-packet buffer is shown in Fig. 6.

In order to compare their aggressiveness against existing congestion controlled flows in the new network, we add a background TFRC flow between R1 and BS1 and let the MN handover from BS2 to BS1. It is easy to see that the bandwidth at BS2 is equal to the fair share at BS1 after the handover. We measure the time the handed-over TFRC flow takes to regain its sending rate and its throughput during this period. Table I shows the simulation results averaged over 15 runs. As expected, the aggressiveness of the enhanced TFRC is in between of the other two, therefore our proposal achieves a better balance between throughput and packet loss.

\section{CONCLUSION}

In this paper we studied the performance of TFRC in a bandwidth-changed handover. When the new bandwidth is lower, heavy packet loss happens before the sender fully slows down. An enhancement to TFRC is proposed that uses selfclocking to limit the sending rate after a handover. At the

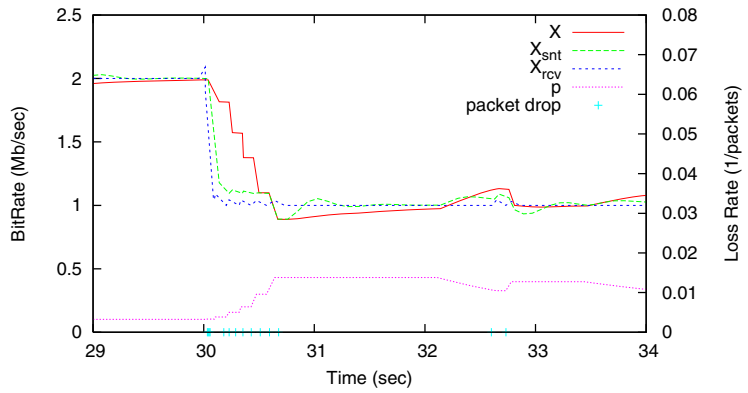

Fig. 5. Enhanced TFRC during a Handover

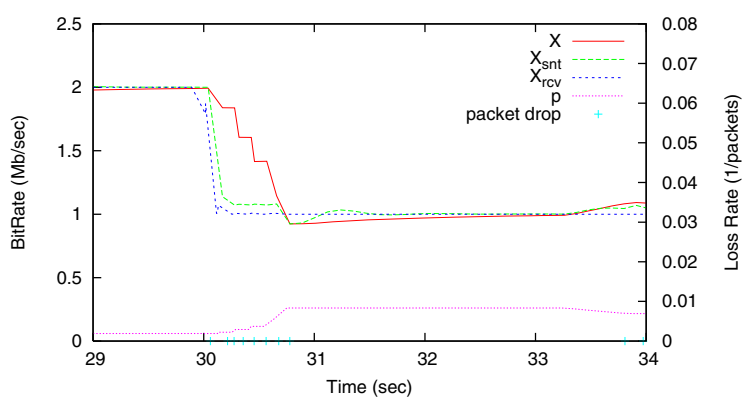

Fig. 6. Smooth TFRC Handover

TABLE I

Simulation Results With Competing TRAFFiC

\begin{tabular}{l||c|c|c}
\hline & Normal TFRC & Conserv. TFRC & Enhanced TFRC \\
\hline Adapt. Time $(\mathrm{sec})$ & 3.09 & 4.59 & 4.26 \\
Throughput $(\mathrm{Mb} / \mathrm{s})$ & 0.76 & 0.65 & 0.71 \\
\hline
\end{tabular}

same time, a more aggressive bandwidth probing mechanism is used for TFRC to compete efficiently for its fair share, compensating the conservativeness of self-clocking. Because lossless handover is not necessarily the optimum for TFRC, in this paper we defined the concept of smooth TFRC handover and discussed its conditions.

\section{REFERENCES}

[1] S. Floyd, M. Handley, J. Padhye and J. Widmer, TCP Friendly Rate Control (TFRC): Protocol Specification, RFC Draft 04. IETF, Jan. 2008.

[2] S. Floyd, M. Handley, J. Padhye and J. Widmer, Equation-Based Congestion Control for Unicast Applications, Proc. ACM SIGCOMM 2000, pp. 43-56, Stockholm, Sweden, August 2000.

[3] J. Padhye, V. Firoiu, D. Towsley and J. Kursoe, Modeling TCP Throughput: A Simple Model and its Empirical Validation, Proc. ACM SIGCOMM 1998, pp. 303-314.

[4] A. Gurtov and J. Korhonen, Measurement and Analysis of TCP-Friendly Rate Control for Vertical Handovers, ACM MCCR 8(3), pp. 73-87, July 2004.

[5] Y. Pan, M. Lee, J. B. Kim and T. Suda, Smooth Handoff Scheme for Stream Media with Bandwidth Disparity in Wireless Cells, Proc. CCW 2003, pp. 9-16, Oct. 2003.

[6] Y. Lin, G. Kuo, H. Wu, Y. Peng and S. Cheng, MBTFRC: A TFRC Enhancement for Heterogeneous Mobile Networks, Proc. IEEE GLOBECOM 2003, vol.5, pp.2880-2884, Dec. 2003.

[7] D. Bansal, H. Balakrishnan, S. Floyd and S. Shenker, Dynamic Behavior of Slowly-Responsive Congestion Control Algorithms, ACM SIGCOMM Computer Communication Review, v.31, n.4, p.263-274, Oct. 2001.

[8] D. Li, K. Sleurs, E. Van Lil, A. Van de Capelle, Fast Link Adaptation for TFRC after a Handover, Proc. IEEE Pimrc'08, Sept. 2008. 